\title{
Die Folgen des EBM-Labor 1999
}

\author{
U. P. Merten und U. Früh
}

\section{Einleitung}

Die Einführung des neuen Einheitlichen Bewertungsmaßstabes (EBM) zum 1. 7. 1999 hat die Laboratoriumsmedizin für die ambulante Versorgung der gesetzlichen Krankenversicherungen (GKV) und der darin versicherten Patienten und Patientinnen erheblich verändert. Vor Einführung des neuen EBM wurde von den Autoren auf die zu erwartenden Folgen dahingehend hingetviesen, da $\beta$ durch die neuen Regelungen ,über $50 \%$ aller heute (1997-1998) für ambulante GKV-Patienten erbrachten Laborleistungen unterbunden werden" [1].

Das Klinische Labor hat einen Anteil von $64 \%$ und die Bakteriologie von $45 \%$ an der ärztlichen Diagnosefindung [2], wobei die Kosten für diese Untersuchungen sowohl in der Klinik als auch ambulant jeweils nur bei insgesamt 2,5-3,2\% der Gesamtkosten der GKV-Ausgaben liegen [3]. Obwohl somit die Laboratoriumsmedizin ein dringend und unverzichtbarer erforderlicher Bestandteil der Diagnosefindung ist und nur einen sehr geringen Anteil an den Gesamtkosten hat, sind an diesen $2-3 \%$ seit Jahren wiederholt extreme Kürzungen vorgenommen worden. Durch den neuen EBM in Verbindung mit der Budgetierung des einsendenden Arztes hinsichtlich OI/II- und speziell der OllI-Laborleistungen wurde eine dramatische Situation erzeugt. Nur durch verschiedene Nachbesserungen durch die Kassenärztliche Bundesvereinigung (KBV) im Jahre 2000 wurde eine Katastrophe verhindert [4].

Die Abrechnungszahlen der Quartale III und IV/1999 bestätigten die von uns 1999 veröffentlichten Befürchtungen [1]. Ergebnisse aus verschiedenen Laboratorien wurden kurzfristig analysiert und in Einzelauswertungen mitgeteilt $[5,6,7,8]$. Diese ersten alarmierenden Notrufe wurden aber mit dem Hinweis, es handele sich um ,nicht repräsentative Einzelerhebungen" abgetan.

Die KBV handelte erst nach Vorliegen der vollständigen Abrechnung für III und IV 1999 und beschloß dann einen linearen Zuschlag von $24 \%$ für die Untersuchungen des Kapitel OIII. Dieser Zuschlag wurde aber von den meisten KVen erst ab I. Quartal 2000 bezahlt, so daß die Laboratorien erhebliche Verluste für das III. und IV. Quartal 1999 zu tragen hatten.

Der 24\% Zuschlag wurde zwar am Ende des Jahres 2000 von der KBV für das I. und II. Quartal 2001 verlängert [9], wobei eine weitere Verlängerung als politisch nicht durchsetzbar erklärt wurde.. Stattdessen soll eine neue Gestaltung und Bewertung des Kapitel $\mathrm{O}$ aufgrund neuer betriebswirtschaftlicher Bewertungen vorgenommen werden.

$\mathrm{Da}$ in der Vergangenheit oft Einzelauswertungen und Einzeldarstellungen als nicht ausreichende Informationsgrundlage angesehen wurden, sollen anhand der vollständigen Abrechnung einer Kassenärztlichen Vereinigung (KV), die Äuswirkungen der letzten Reform aufgezeigt und Anregungen für eine Neubewertung gegeben werden.

\section{Grundlagen}

Als Grundlage der vorliegenden Ausivertung dienen die „Frequenztabellen Vergleichsgruppe“ für Fachgruppe Laborärzte (ambulant) der Quartale III/1998 bis II/2000 einer KV [10], in denen alle für GKV-Patienten/innen erbrachten Leistungen sowohl kurativ als auch für Mutterschafts-Vorsorge, Früherkennung und sonstige Hilfen den jerveiligen Gebühren-Nummern (GO-Nr) und den entsprechenden Kapiteln zugeordnet wurden.

\section{Auswertung}

Es zeigt sich ein überzogener Rückgang der Fallzahlen mit bis zu 29\% bei deutlicher Verminderung der angeforderten Untersuchungen je Fall. Daraus resultiert eine für Patienten wie Laboratorien dramatische Reduzierung der Laboruntersuchungen von $30-46 \%$, zum Teil sogar mehr (über $50 \%$ ) in einzelnen Laboratorien (Tabelle 1).

Nach dem Rückgang der Untersuchungszahlen im OIII-Bereich zeigt sich über die Quartale kaum ein Wiederanstieg, wohingegen die an den Laborarzt überwiesenen Untersuchungszahlen im OI-Bereich nach einem geringen Rückgang wieder auf die Frequenzzahlen vor III/99 zurückgekehrt sind und im I. und II. Quartal 2000 sich sogar gegenüber dem niedrigsten Stand im III. Quartal 1999 verdoppelt haben (Abbildung 1).

Niedergelassene Praxen haben im OIII-Bereich seit dem dritten Quartal 1999 deutlich weniger Untersuchungen angefordert, wobei bestimmte Untersuchungen von vielen Laboratorien nicht mehr angeboten bzw. durchgeführt werden konnten. Diese Untersuchungen sind entweder im EBM primär zu niedrig bewertet oder aber bei der stark abgefallenen Frequenz sind die festgelegten Kostenerstattungssätze im EBM unter die Erstellungskosten gefallen (z. B. für mikroskopische Untersuchungen) (s. u.).

Während sich die Anzahl der Fälle in der Mutterschaftsvorsorge erwartungsgemäl3 nur unwesentlich 
Tabelle 1 Berechnung der kurativen Leisungen pro kurativem Fall

\begin{tabular}{|c|c|c|c|c|}
\hline & Anzahl 3/99 & Anzahl 4/99 & Anzahl 1/00 & Anzahl 2/00 \\
\hline Anzahl Fălle kurativ & 386.457 & 392.431 & 457.705 & 455.914 \\
\hline Anzahl Leistungen kurativ (ohne OI/III) & 1.036 .347 & 1.073 .201 & 1.178 .401 & 1.164 .484 \\
\hline Anzahl kurative Leistungen pro kurativem Fall (ohne OI/II) & 2,7 & 2,7 & 2,6 & 2,6 \\
\hline Rückgang der kurativen Leistungen (ohne $\mathrm{OI} / \mathrm{II}$ ) zum Vorjahresquartal & $-46 \%$ & $-42 \%$ & $-33 \%$ & $-30 \%$ \\
\hline
\end{tabular}

Tabelle 2 Veränderungen zum Vorjahresquartal

\begin{tabular}{lrrrr}
\hline & & Veränderungen zum Vorjahresquartal \\
& Anzahl 3/99 & Anzahl 4/99 & Anzahl 1/00 & Anzahl 2/00 \\
\hline Anzahl Fälle MuVo & $1 \%$ & $3 \%$ & $3 \%$ & $-100 \%$ \\
Anzahl Fälle Früherkennung Männer & $-100 \%$ & $-100 \%$ & $-100 \%$ & $-36 \%$ \\
Anzahl Fälle Früherkennung Frauen & $-1 \%$ & $18 \%$ & $-22 \%$ & $-45 \%$ \\
Anzahl Fälle Früherkennung Kinder & $-7 \%$ & $-40 \%$ & $53 \%$ & $108 \%$ \\
Anzahl Fälle Sonstige Hilfen & $31 \%$ & $38 \%$ & & \\
\end{tabular}

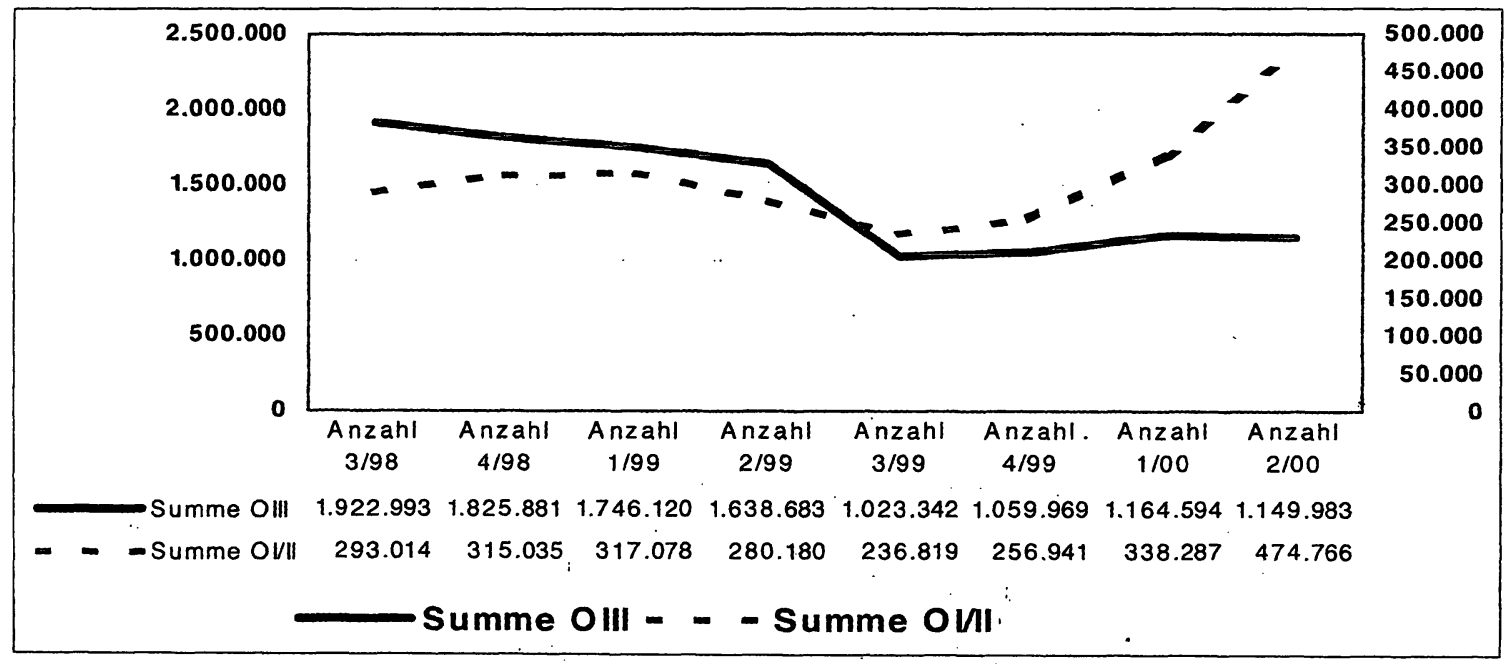

Abbildung $1^{\circ} \mathrm{O} / / 1$ und OIII: Untersuchungsentwicklung (absolute Zahlen)

änderte, sind die Früherkennungsuntersuchungen für Frauen bis zu $36 \%(2 / 00)$ und für Kinder bis zu $45 \%$ $(2 / 00)$ erheblich reduziert worden (Tabelle 2$)$.

Der vorhersehbare Einbruch im III. Quartal 1999 hat sich schon im II. Quartal 1999 mit einem Rückgang der Untersuchungen angekündigt (Abbildung 2). Der dann folgende Abfall der Untersuchungszahlen in den Bereichen Mikroskopie, Bakteriologie, Immunologie und Klinische Chemie ab dem III. Quartal bestätigt die Prognosen der Autoren.

Überweisungen für Untersuchungen des OIII-Bereiches erstrecken sich nicht auf alle OIII-Bereiche gleichförmig, sondern je Bereich und Quartal sind erhebliche Unterschiede festzustellen. Die Rückgänge lagen im III. Quartal 1999 zwischen $29 \%$ und $84 \%$, im IV. Quartal 1999 zwischen 20\% und 59\%, im I. Quartal 2000 zwischen $9 \%$ und $64 \%$ und im II. Quartal 2000 zwischen $13 \%$ und $61 \%$ jeweils gegenüber dem entsprechenden Quartal des Vorjahres (Abbildung 3), wobei wie erwähnt, bereits ab dem II. Quartal 1999 sukzessive weniger angefordert wurde.

Im II. Quartal 1999 waren die molekularbiologischen Untersuchungen mit einem 84\%igen Rückgang am stärksten betroffen, im II. Quartal 2000 sind es die 


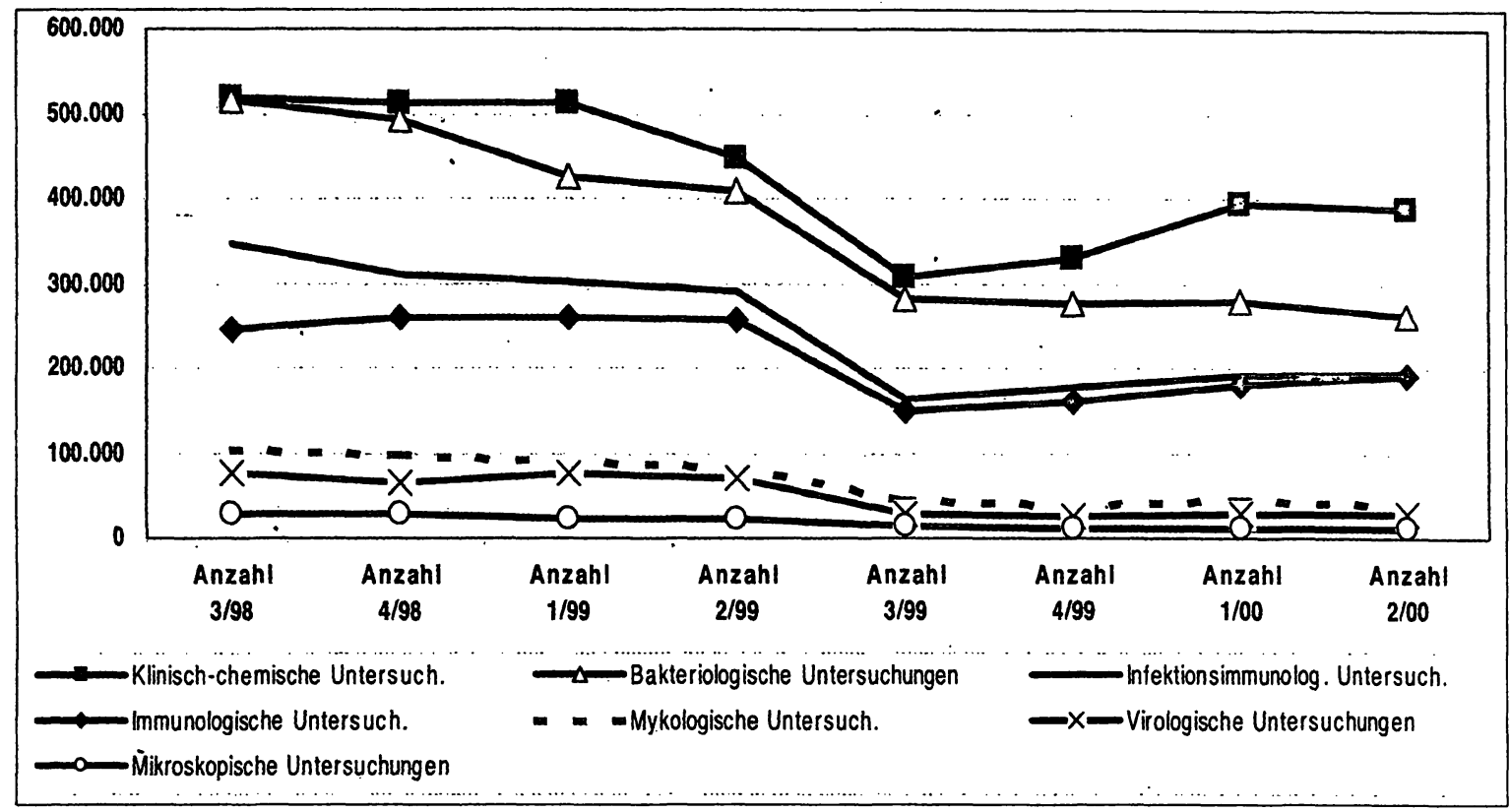

Abbildung 2a Olll-Untersuchungsentwicklung (absolute Zahlen)

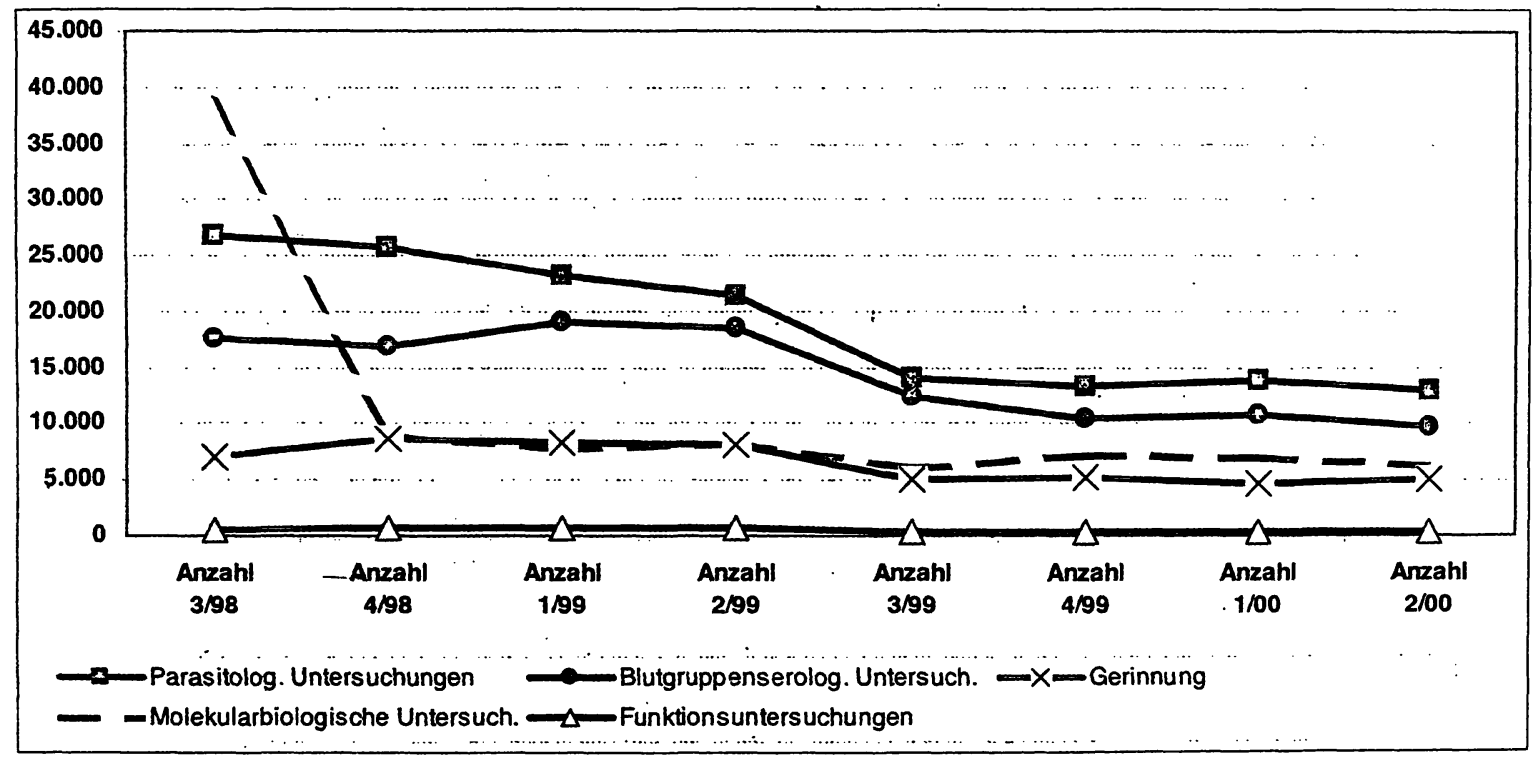

Abbildung 2b Oll-Untersuchungsentwicklung (absolute Zahlen)

virologischen Untersuchungen mit $61 \%$. Rückgang. Untersuchungsfrequenzen für klinisch-chemische, immunologische, infektionsimmunologische und bakteriologische Untersuchungen sind durch die Einführung weiterer Ausnahmeregelungen im Jahr 2000 etwas angestiegen; der Rückgang blutgruppen-serologischer Untersuchungen hat sich jedoch von $-29 \%$ im
III. Quartal 1999 auf $-48 \%$ im II. Quartal 2000 verstärkt.

Mikroskopische Untersuchungen liegen konstant um $51-57 \%$ niedriger als in den Vergleichsquartalen, sie sind systematisch zu niedrig bewertet. Dies trifft auch auf die virologischen Leistungen zu. Bei den derzeitigen Kostenerstattungssätzen unterbleiben unterbe- 


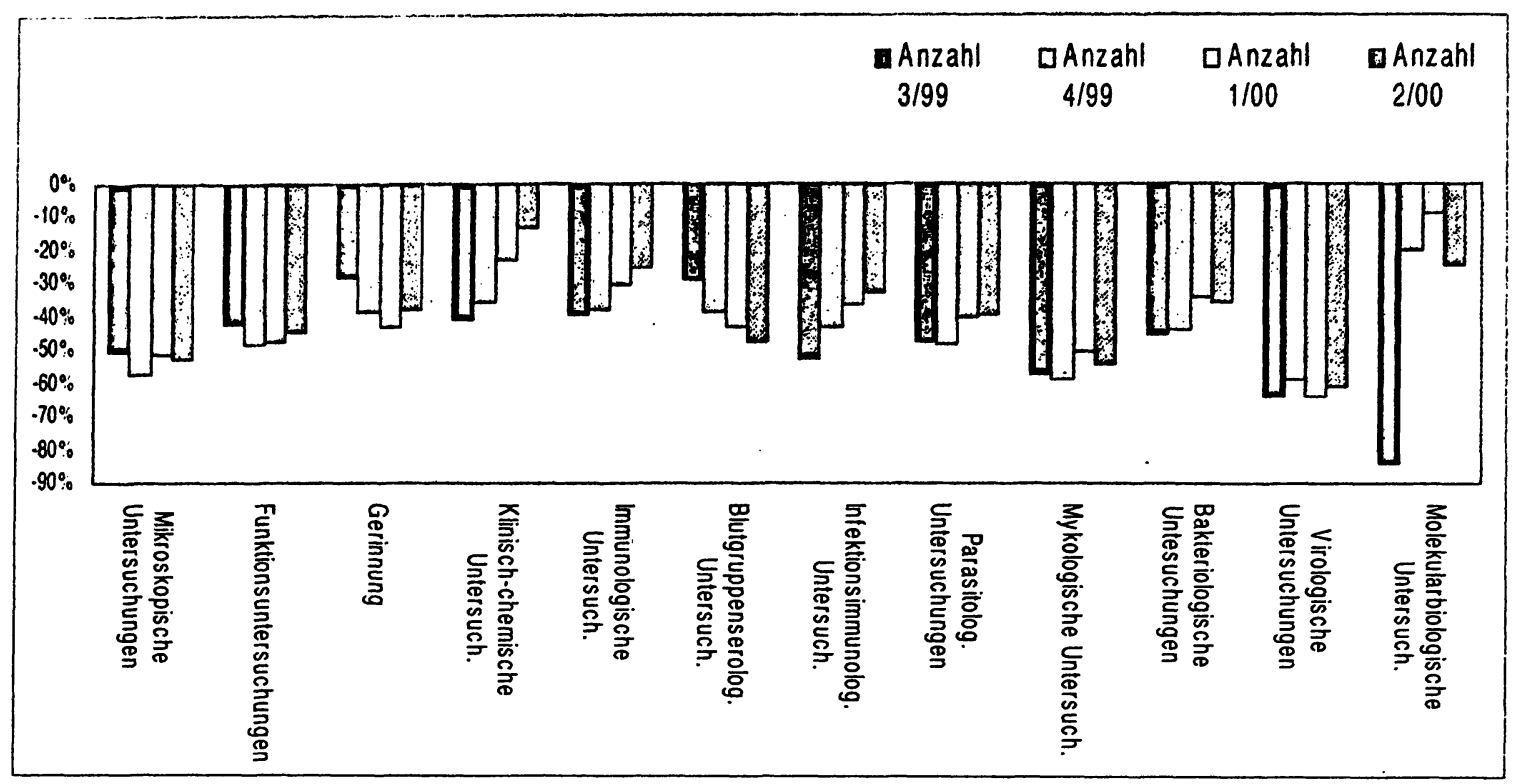

Abbildung 3 OIII-Untersuchungen: \% Veränderung zum Vorjahresquartal

wertete Leistungen bzw. werden auf ein Minimum reduziert, zumal wenn diese, wie mikroskopische und virologische Untersuchungen, mit einem hohen Personalaufwand verbunden sind. Durch eine fachlich gesicherte mikroskopische Untersuchung besteht aber die Möglichkeit einer schnellen patientenorientierten Diagnostik; diese unterbleibt bzw. wird erst gar nicht mehr veranlaßt.

\section{Bewertung}

Die Kostenfindung im EBM 1999 beruht auf einem Gutachten der Firma McKinsey [11] und somit auf Untersuchungsfrequenzen des Jahres 1997. Nach der Einführung des neuen EBM zeigt sich aber ein deutlicher Rückgang aller Untersuchungen und damit eine erhebliche Verkürzung der Serienlängen. Werden Serien kürzer, bewirkt dies eine Kostensteigerung der einzelnen Analyse, die bei kurzen Serien und seltenen Anforderungen oft zu einer Verdoppelung der Kosten führt. Die höheren Kosten der kürzeren Serie müssen in der zu erwartenden Neufestsetzung ausreichend berücksichtigt werden, sonst wird die zur Kostendeckung erforderliche Serienlänge durch Schließen von Regionallaboratorien und Konzentration in Megalaboratorien bewirkt, was zu einem Verlust einer Patienten- und ärztenahen Versorgung mit speziellen Laboruntersuchungen und damit zu einer Gefährdung des Sicherstellungsauftrages der Kassenärztlichen Vereinigung führen wird.

Die derzeitigen Probleme sind auf verschiedene Ursachen zurückzuführen: Häufig wurden Reagenzien-, Geräte- und Personalkosten addiert und damit eine
Kostenfestlegung begründet. Betriebswirtschaftliche Ermittlungen wurden in verschiedenen Laboratorien durchgeführt, ohne ausreichende Berücksichtigung von Regionallaboratorien. Anschließend wurden die erzielten Kosten mit politischen Argumenten weiter abgesenkt. Die realen Erfordernisse und Gegebenheiten werden mit diesen Vorgehensweisen unzureichend berücksichtigt und bewirken, daß die festgelegten Preise im EBM wesentlich zu niedrig liegen, und zusammen mit politisch motivierten Budgets die bestehende Schieflage. Der drastische Rückgang der Laboruntersuchungen war ein weiterer Grund der Fehlentwicklung und zeigt, daß die zugrunde gelegten Prämissen nicht stimmig waren.

\section{Berechnungen}

Die Kostenermittlung ist nicht nur in Laboratorien ein äußerst komplexer Vorgang. Eine Zuordnung von Reagenzien z. T. auch von Gerätekosten über Lieferantenrechnungen ist noch relativ einfach durchzuführen (wobei aber die Auffassung 1 Test = 1 Patientenergebnis falsch ist).

Die Zuordnung von Personalkosten ist mehrfach beschrieben worden (12) und zeigt mit aller Deutlichkeit die Kompliziertheit und die Gefahren eines verkürzten Vorgehens auf. Der Personaleinsatz bei Schnelltests, Einzeltests wie Komplexuntersuchungen ist von dem Personalbedarf bei vollmechanisierten Analysen zu unterscheiden.

Daneben sind die nicht der einzelnen Untersuchung sondern dem Arbeitsplatz als Ganzes zuzuordnenden indirekten Kosten über eine Verteilung auf eine Analysengruppe (z. B. teil- bzw. vollmechanisierte Geräte 
Tabelle 3 Overheadkosten, die allen Untersuchungsparametern durch einen Verteilerschlüssel zugeordnet werden müssen, s. Text

Analytik
Probenverteilungssystem
Kühlräume (z. B. Proben, Reagenzien)
Einrichtung Labor
Lager (Material)
Aufwand Sicherheitslabor
Qualitätssicherung / Qualitätskontrolle
Akkreditierung
Desinfektion
Reparaturdienst
Außendienst

\section{Gesetzliche Auflagen}

Ausfallzeiten / Schwangerschaften

Arbeitsmedizin

Meldungen Infektionsschutzgesetz

Sicherheitsbeauftragte

Strahlenschutzgesetz

Datenschutzbeauftragte

Betriebsrat

Klimatisierung

Entsorgung

\author{
Management \\ Verwaltung/Nersicherungen/Beiträge \\ EDV / Hard- und Software/Reinigung \\ Anmeldung/Befundpapier/Geschäftspapier \\ Telefon - Zentrale \\ Telefon - Auskunft \\ Sekretariat \\ PC-Netz \\ Fortbildung / Literaturdienst \\ Archiv \\ Poststelle \\ Info-Werbung = Informationsdienst \\ Versandmaterial - Transport (PKW etc) - Porto
}

für die Durchführung einer kleineren oder größeren Anzahl von Analysen) zu berücksichtigen. Ein hoher Anteil der realen Kosten liegt in dem sogenannten Overhead, dem Kostenanteil von Untersuchungen, der nicht unmittelbar sondern nur mittelbar zugeordnet werden kann und auf alle durchgeführten Analysen umgelegt bziv. ihnen zugerechnet werden muß $(z$. B. Verwaltung, Abrechnung, Probenverteilungssysteme, Kosten für Qualitätsmanagement, Logistik, LaborEDV, Datenaustausch mit Einsender etc.). Bei bisherigen Kostenermittlungen und Kostendarstellungen ist dieser Kostenbereich oft nicht ausreichend berücksichtigt worden. Seine Bedeutung und vor allem seine Inhalte bedürfen einer systematischen Berücksichtigung.

In Tabelle 3 wird versucht, relevante Kostenstellen des "Overhead" aufzulisten. Zum einen Kosten, die mit der Analytik in Zusammenhang stehen, aber nicht einer einzelnen Analysenserie bzw. Untersuchung zuzuordnen sind; zum anderen Kosten für das Management, für die vom Gesetzgeber geforderten Auflagen und für die Finanzierung der für das Laboratorium erforderlichen Mittel. Sie alle zusammen stellen einen erheblichen Kostenblock dar. Im übrigen ist es erforderlich, auch einen wirtschaftlichen Gewinn für das immense Unternehmensrisiko zu erzielen, ohne den kein Anreiz bestehen kann, Risiko und Verantwortung für einen Laborbetrieb zu übernehmen.
Die Erfahrung zeigt, daß in einem Laboratorium, welches in einem Krankenhaus eingebunden ist und ein begrenztes Spektrum anbietet bzw. selber abarbeitet, der Overheadkostenanteil relativ niedrig ist. Dazu kommt, daß Overheadarbeiten und somit Overheadkosten überwiegend vom „Krankenhausallgemeintopf“ erbracht werden und somit zunächst nicht direkt dem Labor zugerechnet werden. Stationäre Laboratorien sind daher nur eingeschränkt mit niedergelassenen Laboratorien vergleichbar. Freie Laboratorien, die auf dem Markt miteinander konkurrieren und einen größeren Aktionsradius aufweisen, haben im Overheadbereich eine deutlich höhere Belastung. Der Anteil der Overheadkosten zu den Gesamtkosten liegt zwischen 45 und $55 \%$ - teilweise sogar bis $60 \%$. Es zeigt sich, $\mathrm{da} ß$ der prozentuale Anteil der Overheadkosten auch in größeren Laboratorien nicht oder nur unwesentlich günstiger ist als in kleineren Laboratorien. Oligopolartige Laboratorien werden daher nur kurzfristig preiswerter arbeiten, weil sie in den ausgewiesenen Preisen den Overhead nicht oder nur ungenügend berücksichtigen. Die angebotenen Preise sind somit „Werbepreise“, um „Kunden“ zu fangen, sie stellen nicht die realen Kosten dar. Wenn Regionallaboratorien schließen müssen und Megalaboratorien den Markt übernehmen, werden die Preise aller Voraussicht nach erheblich anziehen. 


\section{Fazit}

Dic Kostenerstattungssätze im EBM-Labor 1999 liegen zu niedrig - insbesondere für die Untersuchungen, deren Frequenz über die angestrebten $15 \%$ hinaus abgesunken sind. Bei einer Rücknahme der derzeitigen $24 \%$ igen Anhebung und ohne andere Korrekturen wird ein Sierben der Regionallaboratorien unmittelbar die Folge sein. Gleiches gilt, sollten die z. Zt. bestehenden Ausnahmeindikationen ersatzlos gestrichen werden, da durch einen damit verbundenen erneuten Rückgang der Untersuchungsfrequenzen weitere Analysen deutlich notleidend werden. Die Patientenversorgung wird schlechter und die Diagnostik in die Krankenhäuser transferiert werden. Das Gesamtsystem wird dadurch wesentlich teurer werden.

Bei der anstehenden Neuermittlung und Neufestsetzung der Kostenerstattungssätze für Laboruntersuchungen im Kapitel OIII des EBM ist als Basis eine lineare Anhebung aller Kostensätze erforderlich. Daneben müssen einige Leistungen deutlicher angehoben werden, um eine wirtschaftliche Erbringung zu ermöglichen. Die Kosten einiger Untersuchungen könnten durch Rationalisierungsmaßnahmen weiter reduziert werden, so daß diese auf dem derzeitigem Niveau verbleiben bzw. einige wenige sogar etwas abgesenkt werden können.

Die lineare Anhebung muß mindestens bei $29 \%$ liegen. um die regionale Versorgung der Patienten sicherzustellen. Das System des Wirtschaftlichkeitsbonus muß zugunsten einer intelligenteren Leistungssteuerung, die sich an der medizinischen Notwendigkeit orientiert (z. B. durch Komplexziffern und/oder Leitlinien zur Stufendiagnostik) aufgegeben werden. Komplexziffern können - wo sinnvoll - Einzelleistungsvergütung ergänzen. Höchstwerte können nur für Selbstzuweisungen - nicht bei Fremdzuweisungen - gelten und alle Leistungsbeschreibungen müssen eindeutig definiert werden. Bei Zweifelsfällen bzw. bei Interpretationsdifferenzen darf eine Klärung bzw. Auslegung nur durch eine fachkompetente Laborkommission erfolgen. Dies betrifft auch die Definition, was und wie Untersuchungen als „Ähnliche Verfahren“ abgerechnet werden sollen. Obsolete Verfahren müssen von einer Laborkommission aus dem EBM gestrichen und neue Verfahren zeitnah in den EBM aufgenommen werden, wobei entsprechende Gelder und damit eine entsprechende Kostenabdeckung und Honorarzahlung von den Kassen jeweils bereit gestellt werden müssen.
Die ambulante Versorgung der gesetzlich versicherten Patienten und Patientinnen ist auf einer absoluten Talsohle angekommen. Die „Unterbindung der Koppelungsgeschäfte" ist nicht gelungen und das "Problem Laborgemeinschaften" muß neu diskutiert werden. Die KBV hat aber den weiteren Reformbedarf erkannt. Kritiker müssen ernst genommen werden, die es für unethisch halten, daß Àrzte dafür finanziell belohnt werden, daß sie Leistungen nicht erbringen bzw. nicht veranlassen [11].

In die Neufassung des Kapitels $O$ im EBM liegt eine hohe Verantwortung. Es kann nicht mehr um eine Honorarabsenkung oder Honorarfreisetzung gehen. Es geht um den Erhalt einer Patienten-/und Arzt-nahen Versorgung mit speziellen Laboruntersuchungen und damit um den Bestand des Sicherstellungsauftrages der Kassenärztlichen Vereinigung.

\section{Literatur}

1. U. P. Merten und U. Früh; Der Internist 1999;3:M70-M73.

2. J. D. Kruse-Jarres; Labmed 1994;18:213-219.

3. Quelle: Bundesminister für Gesundheit / Verband der Diagnostica Industrie (VDGH).

4. KBV, Veröffentlichung, Deutsches Ärzteblatt (2000).

5. P. H. Rodt - persönliche Mitteilung (1999). Vortrag Entwicklung und Folgen der Laborreform, Herbsttagung des Berufsverbandes Deutscher Laborärzte, Berlin,.September 2000.

6. R. Latza - persönliche Mitteilung (1999) und Vortrag Herbsttagung des Berufsverbandes Deutscher Laborärzte, Berlin, September 2000 .

7. B. Heicke - Vortrag Herbsttagung des Berufsverbandes Deutscher Laborärzte, Berlin, September 2000.

8. A. Bobrowski - Vortrag Vertreterversammlung der $\mathrm{KVSH}, \mathrm{Ok}-$ tober 2000, persönliche Mitteilung.

9. KBV, Veröffentlichung, Deutsches Ärzteblatt (2000).

10. Übersichten Frequenztabelle Vergleichsgruppe für Laborärzte (ambulant) der Kassenärztlichen Vereinigung.

11. Kassenärztliche Bundesvereinigung, McKinsey-Analyse, April 1998.

12. K. Osburg: Personalbedarf und Kosten in medizinischen Laboratorien; INSTAND Schriftenreihe Bd. 1, 3. Auflage, Springer Verlag Berlin (1987)

13. M. Richter-Reichhelm, Medical Tribune, 35: Nr. 38, 22. September 2000.

Dr. med. Utz P. Merten

Facharzt für Laboratoriumsmedizin,

Mikrobiologie und Infektionsepidemiologie

Stadtwaldgürtel 35

50935 Köln

Uli Früh, Dipl. Betriebswirt (FH)

Obere Wässere 1

72764 Reutlingen 